Abanico Veterinario. Enero-Diciembre 2020; 10:1-19. http://dx.doi.org/10.21929/abavet2020.13 Artículo Original. Recibido: 11/04/2020. Aceptado: 03/07/2020. Publicado: 15/07/2020.

\title{
La organización y agrupación como eje toral para el diseño de esquemas de atención a caprinocultores en el norte de México: Estudio de caso
}

\author{
Organization and grouping as a key axis to design attention schemes for goat \\ farmers in northern Mexico: Case study
}

\section{Jesus Ramos-Martinez ${ }^{1}$ ID, Homero Salinas-González ${ }^{1,2}$ ID, Manuel Medina- Elizondo $^{1 \mathrm{ID}}$, Uriel Figueroa-Viramontes ${ }^{3 \mathrm{ID}}$, Jorge Maldonado-Jáquez ${ }^{\star 3 \mathrm{ID}}$}

\begin{abstract}
${ }^{1}$ Programa de Posgrado. Universidad Autónoma de Coahuila, Facultad de Contaduría y Administración. Torreón, Coahuila, México. ${ }^{2}$ Lincoln University of Missouri. Lincoln University Cooperative ExtensionSmall Ruminant Program. Jefferson City, Missouri. USA. ${ }^{3}$ Instituto Nacional de Investigaciones, Forestales, Agrícolas y Pecuarias. Campo Experimental La Laguna. Matamoros, Coahuila, México. *Autor para correspondencia: Jorge Maldonado-Jáquez. Campo Experimental La Laguna. Boulevard José Santos Valdez 1200 Pte. C.P. 27440, Matamoros, Coahuila, México. jesusrmz02@gmail.com, homero.salinas@hotmail.com, drmanuelmedina@yahoo.com.mx, figueroa.uriel@inifap.gob.mx,
\end{abstract} maldonadoj.jorge@hotmail.com

\section{Resumen}

El objetivo del presente estudio, fue organizar y agrupar caprinocultores con perfiles socioeconómicos y tecnológicos similares, con la finalidad de diseñar esquemas de atención dirigida y acorde a sus necesidades. El estudio constó de dos fases: En la primera se estratificó, delimitó y caracterizó el perfil socioeconómico y productivo de los caprinocultores. La segunda fase se realizó considerando la percepción de los productores hacia los principales problemas y limitantes del sistema de producción. Se realizó un análisis de correspondencia múltiple. Se identificaron 5 grupos de productores. G1 presenta la mayor producción de leche $\mathrm{d}^{-1}(\mathrm{p}=0.03)$, el mayor número de hectáreas susceptibles a cultivo y hectáreas sembradas $(p<0.0001)$. G2 posee el mayor nivel educativo $(p=0.0002)$. G3 presenta mayor edad $(p<0.0001)$, mayor número de hijos $(p<0.0001)$ y precio de comercialización (sin diferencia). G4 tiene la mayor edad ( $p=0.0001)$ y tiempo en la actividad ( $p=0.0011)$, y $G 5$ mayor número de hijos $(p=0.0001)$, tamaño de rebaño $(p=0.03)$ y horas dedicadas al pastoreo $(p=0.02)$. El análisis de componentes principales indicó que los CP 1 y CP 2 presentan el $46.55 \%$ de la variación. Los productores refirieron los principales problemas que limitan la productividad. La estratificación y agrupación de productores basados en sus similitudes permite definir claramente puntos críticos de atención, de tal manera que las políticas de fortalecimiento hacia estos grupos marginados cumplirán más fácilmente su cometido.

Palabras Clave: caprinocultores, organización, agrupación, problemáticas comunes y acciones estratégicas.

Abstract
The present study aimed to organize and group goat farmers with similar socioeconomic and
technological profiles to design targeted attention schemes according to their needs. The study
consisted of two phases: As for the first phase, the socioeconomic and productive profile of the
producers were stratified and characterized. In the second phase, goat producers' perceptions were
considered to carry out the main problems and limitations of the production system. The data were
analyzed through Multiple Correspondence Analysis. As a result, five groups of producers were
identified: $G 1$ with the highest milk production $d-1(p=0.03)$, the highest number of hectares susceptible
to cultivation, and with the hectares sown ( $p<0.0001)$; $G 2$ with the highest educational level $(p=0.0002)$;
G3 compounded by elder people ( $<0.0001)$, with a great number of children $(p<0.0001)$, and without a
difference in commercialization price; $G 4$ compounded by elder people $(p=0.0001)$ with inactivity time
( $p=0.0011)$, and, G5 with the highest number of children ( $p=0.0001)$, the highest flock size $(p=0.03)$ with
more hours dedicated to grazing $(p=0.02)$. The analysis of Principal Components indicated, that, CP 1


and CP 2, presented the highest proportion of the variation (46.55\%). Additionally, producers reported the main problems that limit their productivity. The stratification and grouping of goat farmers based on their similarities allowed defining critical points of attention, in such a way that strengthening policies towards these marginalized groups will easily fulfill their mission.

Keywords: goats, extensive system, technology transfer.

\section{INTRODUCCIÓN}

Para cualquier país, las empresas agropecuarias juegan un rol muy importante, ya que son unidades económicas que proveen alimentos para la población (GarcíaPérez, 2017). En este sentido, la empresa agropecuaria combina conocimientos, información, recursos naturales, humanos y de capital para producir bienes de origen animal o vegetal; o servicios para un mercado objetivo y dentro de una operación rentable y sostenible (Guerra, 1998). Sin embargo, muchas de estas empresas agropecuarias son pequeñas o medianas e independientemente de su perfil productivo; se caracterizan por poseer recursos naturales limitados y por presentar constantemente déficit de capital para optimizar las actividades de producción y poca o nula administración (Balestri et al., 2001). Por lo anterior, Aguilar et al. (2016), señalan que la mejora dentro de estas empresas y sin importar el tamaño de la unidad de producción, se dará cuando se ponga en marcha una administración profesional, ya que es cuando se considerará la evolución de las teorías administrativas para mejorar la rentabilidad de las empresas.

Por otra parte, la producción de pequeños rumiantes es muy importante en la ganadería de todo el mundo, específicamente los productos lácteos de origen caprino, los cuales tienen un importante impacto social en áreas de suma pobreza (Dubeuf, 2005; Escareño et al., 2012; Salinas-González et al., 2015), y en determinadas regiones resultan de gran interés por ser un recurso sostenible y muy rentable (Sanz et al., 2003), ya que de su producción deriva la producción de alimentos, contribuyen al empleo y la estabilidad económica, generan valor adicional en las unidades de producción y facilitan el uso de tierras marginales (Escareño et al., 2011); pero tienen la limitante de que son administradas directa o indirectamente por el productor (Cofré, 2001) y la mayoría de las veces, éstos carecen de experiencia en administración.

En México, la caprinocultura representa una actividad que mantiene a casi 1.5 millones de personas y está asociada a estratos de población rural con menores ingresos, ya que representa el sustento para el $80 \%$ de los productores de subsistencia y se desarrolla principalmente en regiones áridas y semiáridas; las cuales corresponden al $60 \%$ del territorio nacional, en donde abundan agostaderos con limitados recursos naturales y zonas con alto grado de marginación (Guerrero-Cruz, 2010; SAGARPA, 2007). Asimismo, se sabe que la producción de leche de cabra representa una fuente importante de empleo para las familias en las zonas rurales, ya que ofrece estabilidad laboral (Escareño et al., 2011).

En este sentido, la Comarca Lagunera, al norte de México, es considerada como la principal cuenca de producción de leche de cabra; sin embargo, se ha documentado que aunque es la principal región productora de leche, aún se presenta una baja productividad; dado principalmente por la falta de registros productivos, nula organización de productores para la producción, procesamiento y/o comercialización 
de leche y carencia de información y asistencia técnica; lo cual deriva en un bajo nivel tecnológico por lo que, mientras no se visualice como empresa, difícilmente la actividad caprina prosperará como negocio (Maldonado-Jáquez et al., 2018) y seguirá siendo solo una actividad de subsistencia. No obstante, se han realizado diferentes esfuerzos de investigación, pero estos no han tenido el suficiente impacto en la innovación de los sistemas de producción (Salinas-González et al., 2016), debido principalmente a la falta de tecnologías adaptadas a las condiciones y características de cada productor.

Por lo anterior, en la búsqueda de soluciones a la problemática que presentan los productores de la Comarca Lagunera, Gómez (2007), se sugiere a la organización como una alternativa para mejorar la rentabilidad de la actividad, ya que dentro de un grupo cooperante y organizado no se dependería totalmente de intermediarios, el precio de la leche se podría negociar y se optimizarían los procesos participativos en los que se busque encontrar áreas de oportunidad para la innovación y eficiencia en el uso de los recursos del productor (Salinas-González et al., 2011).

Por lo tanto, el objetivo del presente estudio fue caracterizar y agrupar productores con perfiles socioeconómicos y tecnológicos similares en la porción noreste de la región Lagunera, en el estado de Coahuila, México; con la finalidad de diseñar programas y acciones estratégicas para la investigación, innovación, transferencia de tecnología y/o capacitación, acordes a sus necesidades; lo cual permita mejorar el sistema productivo, bajo el supuesto de que al transitar a formas cooperadas de producción y comercialización, se incrementará la adopción de tecnologías y en consecuencia la rentabilidad de las empresas.

\section{MATERIAL Y MÉTODOS}

La investigación se desarrolló en la porción noreste de la Región Lagunera, en el estado de Coahuila, que comprende a los municipios de Francisco I. Madero, San Pedro de las Colonias y Matamoros. El clima para los municipios de Francisco I. Madero y San Pedro de las Colonias es subtipo seco semi cálido y el clima para el municipio de Matamoros, es muy seco y cálido. La temperatura media anual ronda entre los $20^{\circ} \mathrm{C}$ y $24^{\circ} \mathrm{C}$, con precipitación media anual entre 200 a $400 \mathrm{~mm}$ (INAFED, 2010).

Se tomó como estudio de caso a productores de la Cooperativa de Reconversión Productiva La Laguna, S.C. de R.L. de C.V. de reciente formación (2016); la cual agrupa a 32 productores, después de que se visitaron 14 ejidos y se invitó a más de 300 caprinocultores del área de estudio.

El estudio constó de dos fases; en la primera se entrevistó a los 32 productores agremiados a la Cooperativa de Reconversión Productiva, misma que se dedica a la producción de leche de cabra en los municipios antes mencionados. Este estudio se realizó con la finalidad de estratificar y caracterizar el perfil socioeconómico y productivo de los productores. La recolección de datos se realizó mediante entrevista directa y visitas a las unidades de producción. En este estudio se utilizaron los métodos de observación, entrevista directa e historias de vida para recabar y 
documentar información de la cadena de producción y comercialización de la leche de cabra (Hernández-Sampieri et al., 2006).

El cuestionario se elaboró con base en la revisión de literatura especializada y de encuestas de diagnóstico, propuesta por SAGARPA (2015). La entrevista se organizó en 13 bloques, en donde se consideraron 44 variables ordinales y nominales. La información obtenida fue capturada en una matriz del programa Excel, y posteriormente los datos fueron analizados mediante un análisis de correspondencia múltiple; el cual se realizó utilizando los paquetes estadísticos InfoStat (Balzarini et al., 2008) y SPSS, adaptando la metodología descrita por Bernal-García et al. (2003), Carrasco-González (2004) y Castel et al. (2003), en el análisis de estadística multivariante.

Para el análisis de correspondencia múltiple se eligieron variables duras de características socioeconómicas, de producción y comercialización; las cuales fueron consideradas como variables de calidad, de acuerdo a estudios previos realizados por Salinas-González et al. (2016), Escareño et al. (2011) y Castel et al. (2003); entre las que se consideraron: edad, escolaridad del productor, dependientes económicos e integrantes de la familia, tiempo dedicado a la actividad, tamaño del rebaño, horas dedicadas al pastoreo, producción de leche y precio, entre otras; las cuales fueron tomadas como la base en los análisis de tipo descriptivo, de Conglomerados por Agrupación Jerárquica (ACJ), Componentes Principales (ACP) y de Correspondencia Múltiple (ACM).

Para este caso se consideró este tipo de análisis, ya que disminuye el rango de covarianza dado por la sobre parametrización, cuando el número de características es muy amplio; reduce la dimensionalidad de las características correlacionadas y genera un reducido número de variables. Posteriormente para realizar el agrupamiento, se parte de una matriz de distancias, conteniendo todas las distancias entre pares de objetos y se agrupan considerando la mínima distancia (Peña-Malavera et al., 2010; Agudelo-Gómez et al., 2015).

La segunda fase del estudio comprendió talleres participativos, en donde a los productores previamente entrevistados se les presentaron los resultados de las encuestas aplicadas en la primera fase. Los talleres tuvieron la finalidad de que los productores formularan los principales problemas y limitantes del sistema de producción, así como propuestas de solución; para ello se empleó la técnica cualitativa de estudio Focus Group (Santiago y Roussos, 2010). Esta metodología es una técnica de recolección de datos que utiliza una encuesta grupal semiestructurada, dirigida hacia una temática específica y de la que el conocimiento existente es inadecuado o es muy complejo para hacer inferencias sin considerar al usuario o grupo de interés (Escobar y Bonilla-Jiménez, 2009).

Los resultados obtenidos se clasificaron y agruparon de acuerdo a la mayor frecuencia, y partiendo de aquí, se elaboró un árbol de problemas para ayudar a identificar los principales problemas y su solución, a través de las causas raíz y efectos (Hernández-Hernández y Garnica-González, 2015); donde en el despliegue estratégico, el problema principal se convirtió en el objetivo estratégico y las causas 
raíz en las acciones estratégicas a implantar; esto se realizó para los productores de cada conglomerado encontrado.

\section{RESULTADOS Y DISCUSIÓN}

Los grupos de productores, aún los organizados como es el caso de la Cooperativa de reciente formación, están conformados por individuos y unidades de producción de diversas características. Al agrupar a los integrantes de los sujetos de intervención tecnológica, se pueden formular estrategias asertivas, ya sea por la gestión de la innovación o las actividades básicas de transferencia de tecnología y capacitación. Los resultados encontrados muestran diferencias suficientes entre subgrupos (conglomerados), por lo que se presentan estrategias específicas a implementar dentro de la cooperativa, según el subgrupo de participantes. Asimismo, se discuten el diseño e implantación de ciertas acciones estratégicas dentro del plan de desarrollo de la Cooperativa.

\section{Variables descriptivas}

En la tabla 1 se muestran las variables descriptivas, en general de los productores para esta región. Al respecto, la literatura que describa las características de los caprinocultores en la Región Lagunera es limitada, y la investigación se ha centrado mayormente en caracterizar la productividad del ganado caprino (Torres-Hernández et al., 2016); es aquí donde radica la importancia de describir este componente del sistema de producción. Se encontró que las edades encontradas para los caprinocultores de la región son menores a las reportadas por Salinas-González et al. (2016), en una región vecina a la de este estudio (suroeste del estado de Coahuila). Además, respecto a la escolaridad encontrada, esta es similar a la reportada por AlvaPérez et al. (2018) y Chipasha et al. (2017), para productores del Altiplano Tamaulipeco en México y el Distrito de Choma en Zambia.

Tabla 1. Variables descriptivas de caprinocultores del noreste de la Región Lagunera en el norte de México

\begin{tabular}{lr}
\hline \multicolumn{1}{c}{ Variable } & Media \pm D.E. \\
\hline Edad (Años) & $50.31 \pm 14.98$ \\
Escolaridad (Años) & $1.47 \pm 1.05$ \\
Número de hijos & $2.94 \pm 1.81$ \\
Número de dependientes & $3.47 \pm 1.78$ \\
Años en el domicilio & $29.16 \pm 19.64$ \\
Años en la actividad & $27.38 \pm 16.52$ \\
Número de cabras & $84.53 \pm 39.44$ \\
Número de sementales & $2.03 \pm 1.15$ \\
Horas de pastoreo (h/día) & $7.44 \pm 2.66$ \\
Litros de leche (L/animal/día) & $1.49 \pm 0.61$ \\
Precio por litro de leche* & $0.24 \pm 0.03$ \\
Hectáreas totales & $2.47 \pm 3.03$ \\
Hectáreas sembradas & $1.13 \pm 1.87$ \\
\hline
\end{tabular}

D.E.= Desviación estándar. *Precio por tipo de cambio de $\$ 1.00$ dólares americanos por $\$ 19.49$ MXN al 8 de mayo de 2019).

Por otra parte, el tiempo viviendo en el mismo domicilio, se le denomina arraigo, y habla de la permanencia de los productores en las localidades de estudio; los años en 
la actividad refieren a la experiencia que tienen los productores, la cual se puede considerar para describir algunas características importantes en el sistema de producción; por ejemplo, la adopción de tecnologías e innovaciones por parte de los productores. Al respecto, Alva-Pérez et al., (2018); Chipasha et al. (2017) y Fonseka et al. (2018a), indican un promedio de 10, 9.2 y 10 años en la actividad de los productores de Tamaulipas, México, Zambia y Sri Lanka, respectivamente; lo cual es inferior al tiempo en la actividad de los productores de este estudio.

En cuanto a las variables productivas, la información sobre tamaño del rebaño, producción de leche y horas que dedican al pastoreo, se pueden utilizar como indicadores de productividad. Al respecto, el dato de producción reportado por los productores en este estudio, es superior al reportado en otros trabajos en regiones vecinas. Maldonado-Jáquez et al. (2018), señalan una producción promedio en cabras locales del suroeste del estado de Coahuila, México, dentro de la Comarca Lagunera, del orden de $938 \mathrm{~g}$. animal ${ }^{-1}$. día ${ }^{-1}$. Asimismo, el precio por litro de leche al que normalmente venden los productores, es el principal factor de incidencia sobre la rentabilidad de la actividad.

Finalmente, la tenencia de tierras, así como el número de hectáreas totales que poseen y el número de hectáreas que siembran, es indicativo de la posibilidad de producir o no forrajes $u$ otros cultivos que pudieran utilizar, ya sea para alimentar al ganado o bien para obtener ingresos extras y completar el ingreso familiar. En este sentido, Mendoza-Jiménez y Ortega-Sánchez, (2009), reportan que aunque el 100\% de los productores pertenecen al sistema de producción extensivo, comercializan sus productos; principalmente carne, en localidades vecinas a precios que impone el recolector; de manera muy similar a los resultados de nuestro estudio, lo cual indica que la comercialización es un problema que no solamente afecta a los productores del norte del país.

\section{Análisis de Componentes Principales}

El ACP (tabla 2), indicó que los CP 1 y 2 explican la mayor proporción de la variación (28.51 y $18.04 \%$, respectivamente). Al respecto, el CP 1 se caracteriza por considerar aspectos socioeconómicos y de producción, e incluye las siguientes variables ordenadas por importancia: edad y escolaridad del productor, años en la actividad caprina (experiencia), litros de leche por cabra-1 $\mathrm{dia}^{-1}$, hectáreas de terreno sembradas y está asociado positivamente con la edad (0.8592) y años en la actividad caprina (0.6502); pero también está asociado negativamente con educación $(-0.7613)$, litros de leche por cabra ${ }^{-1}$ dia $^{-1}(-0.6432)$ y hectáreas sembradas (-0.5204). Por lo que el CP 1 representa a los productores de mayor edad, con más años en la actividad caprina, con menor educación, con menor producción de leche y con menor superficie cultivada.

EI CP 2 incluye únicamente la variable número de cabras. El resto de los componentes están asociados en menor proporción con las variables estudiadas. Por lo anterior, estos dos componentes fueron los únicos que se incluyeron en el ACM. Al respecto, la composición del CP1 y CP2 referencian adecuadamente las características que presentan los caprinocultores del sistema extensivo de México, específicamente para las zonas áridas y semiáridas del norte del país, pues la caprinocultura es la única 
actividad que genera ingreso y alimento para familias en áreas marginadas (BarreraPerales et al., 2018).

Tabla 2. Proporción acumulada de la variación en el Análisis de Componentes Principales para la agrupación de componentes considerados en el Análisis de Correspondencia Múltiple

\begin{tabular}{lllll}
\hline Componente & Autovalor & Diferencia & $\begin{array}{l}\text { Proporción de la } \\
\text { variación }\end{array}$ & $\begin{array}{l}\text { Proporción } \\
\text { acumulada }\end{array}$ \\
\hline 1 & 2.8508 & 1.0468 & 0.2851 & 0.3 \\
2 & 1.8039 & 0.6132 & 0.1804 & 0.5 \\
3 & 1.1907 & 0.1345 & 0.1191 & 0.6 \\
4 & 1.0562 & 0.1988 & 0.1056 & 0.7 \\
5 & 0.8574 & 0.1361 & 0.0857 & 0.8 \\
6 & 0.7214 & 0.1721 & 0.0721 & 0.8 \\
7 & 0.5493 & 0.0814 & 0.0549 & 0.9 \\
8 & 0.4679 & 0.1477 & 0.0468 & 0.9 \\
9 & 0.3202 & 0.1382 & 0.0320 & 1.0 \\
10 & 0.1820 & & 0.0182 & 1.0 \\
\hline
\end{tabular}

\section{Conglomerados por Agrupación Jerárquica}

En la figura 1, se muestra la formación de los conglomerados (grupos), así como las distancias entre ellos. El análisis ubicó a los productores que contenían algún tipo de semejanza en conglomerados, sintetizando y aprovechado la información disponible, y teniendo como resultado un conjunto de información agrupada según su nivel jerárquico y en el que se definieron 5 grupos de conglomerados.

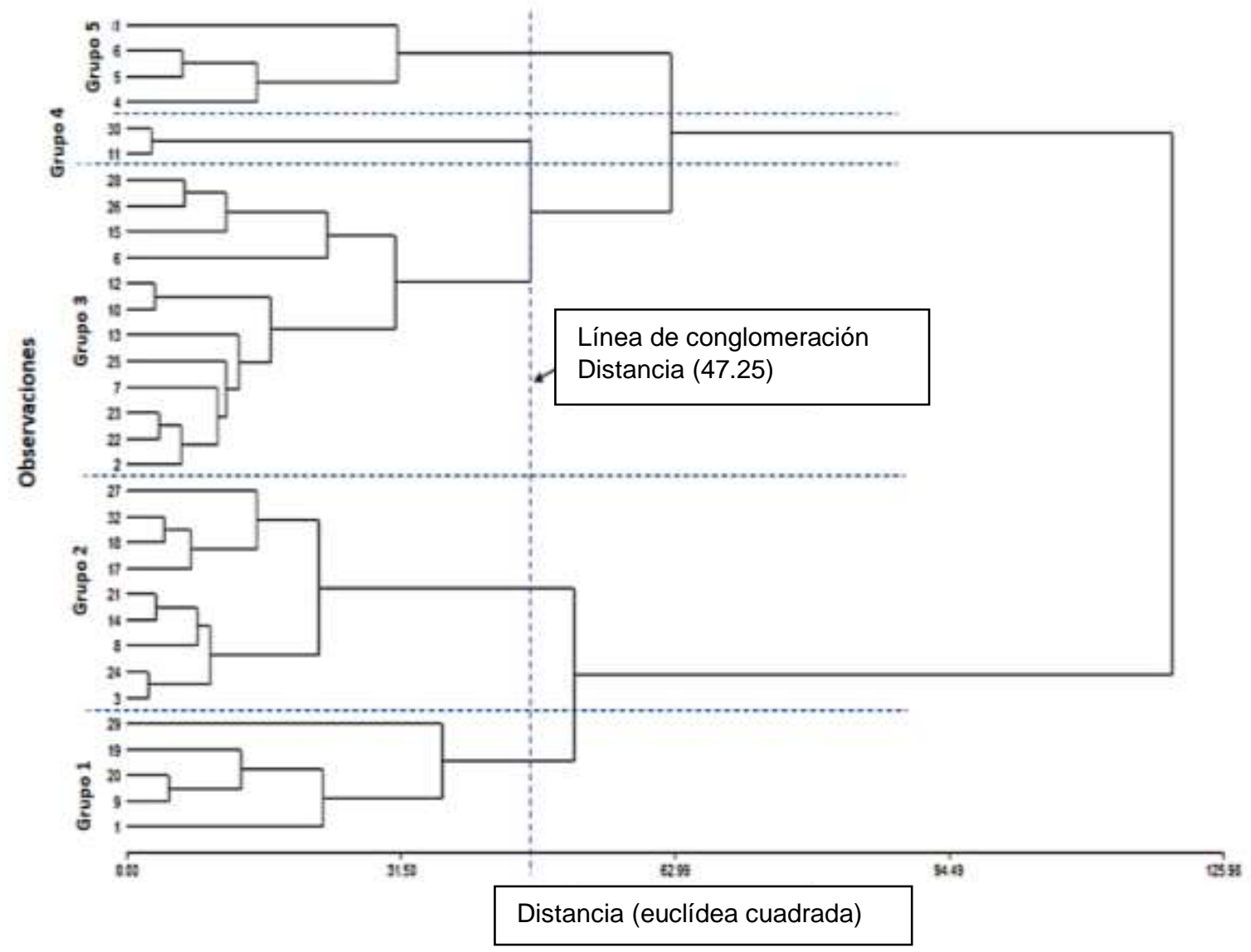

Figura 1. Dendograma del análisis de conglomerados jerárquicos por el método Ward 


\section{Análisis de Correspondencia Múltiple}

En la figura 2, se muestran las interacciones entre los resultados de ACP y ACJ; se tomaron como factores los CP 1 y CP 2, ya que son los que presentan mayor varianza en la toma del conjunto de datos y variables ( 0.2851 y 0.1804 respectivamente; tabla 2). Aquí se pueden observar los resultados del perfil para los productores de cada grupo y considerando que un porcentaje muy bajo de los productores han recibido algún tipo de capacitación, esto servirá para establecer las directrices a seguir para la implementación de esquemas de transferencia de tecnología, así como de innovación e investigación en cada grupo.

Respecto de las interacciones observadas en la figura 2, los productores del grupo 1, se caracterizan por tener la mayor producción de leche en litros al día, superficie total y superficie sembrada; lo cual muestra un área de oportunidad en la mejora de aspectos productivos, pero también una ventana de oportunidad considerable en cuanto a aspectos socioeconómicos.

Por otra parte, los productores del grupo 2 cuentan con el mayor nivel educativo, lo cual puede considerarse como un área de oportunidad para una mayor incorporación de tecnologías en aspectos técnico-productivos. Lo anterior coincide con el reporte de Hundal et al. (2016), en donde indican que productores con cierto grado de entrenamiento, mejoran su conocimiento en la actividad significativamente más rápido que los demás productores. Los productores del grupo 3, presentan una ventana de oportunidad en aspectos productivos, además de que son uno de los grupos con mayor edad y obtienen el mayor precio por litro de leche; por lo que en este grupo resultaría relativamente sencillo aplicar un programa de transferencia de tecnología, ya que carecen de capacitación en todos los eslabones de la cadena productiva. Al respecto, Chipasha et al. (2017), agrupan a productores, los cuales tienen pocas habilidades de producción, poco acceso a mercado e infraestructura y alta incidencia de enfermedades, y los señalan como el grupo con mayores limitantes de producción y áreas de mejora perfectamente delimitadas.

Respecto a los productores del grupo 4, estos se caracterizan por ser de edad avanzada, tener el mayor número de dependientes, más experiencia en la actividad caprina y mayor arraigo en la localidad. Por otra parte, los productores que comprenden el grupo 5, tienen un mayor número de hijos, mayor número de cabras y sementales, con más horas dedicadas al pastoreo; por lo que están mayormente relacionados con aspectos productivos, pero también se incluye el número de hijos; lo cual puede considerarse como una ventaja para establecer algún esquema de transferencia de tecnología, puesto que pueden tener acceso a una mayor cantidad de mano de obra, en comparación con el resto de los grupos. 


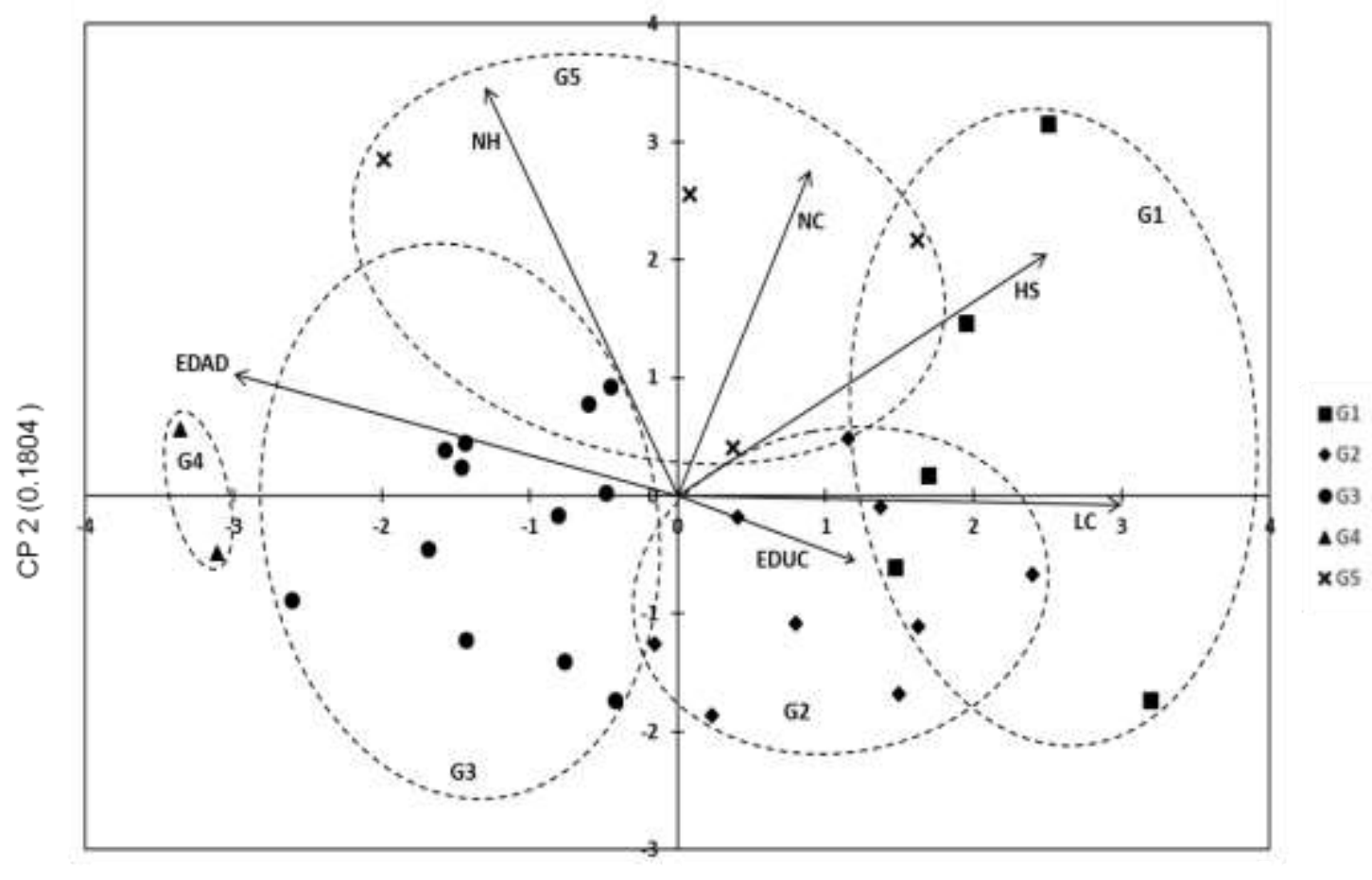

CP $1(0.2851)$

Figura 2. Conjunto de los $\mathrm{CP} 1$ y $\mathrm{CP} 2$ en donde se integran como factores. $\mathrm{NH}=\mathrm{Número}$ de hijos; $\mathrm{NC}=$ Número de cabras; $H S=$ Hectáreas sembradas; $L C=$ litros de leche cabra ${ }^{-1} \mathrm{dia}^{-1}$; $E D U C=$ Escolaridad; G1, G2, G3, G4 y G5= Grupos resultantes del análisis de ACJ

\section{Análisis de Estadísticas Descriptivas por Grupo Conglomerado}

En la tabla 3, se presentan las diferencias entre las medias de las variables entre los 5 conglomerados. El grupo 1 refiere tener la mayor producción de leche $d^{-1}(p=0.03)$, mayor número de hectáreas totales y hectáreas sembradas $(p<0.0001)$. El grupo 2 posee el mayor nivel educativo $(\mathrm{p}=0.0002)$ y una característica sobresaliente de este grupo es que mayoritariamente (57\%) el jefe de familia es mujer; esta información difiere del reporte de Alva-Pérez et al. (2018), ya que para el altiplano Tamaulipeco en México, únicamente el $4 \%$ de las unidades de producción son manejadas por mujeres. A su vez, el grupo 3 es uno de los grupos con mayor edad ( $p<0.0001)$, mayor número de hijos $(p<0.0001)$ y el mayor precio de comercialización (sin diferencia estadística entre grupos); el grupo 4 presenta la mayor edad ( $p=0.0001$ ), y experiencia en la actividad caprina $(p=0.0011)$. Finalmente, el grupo 5 posee una mayor edad $(p<0.0001)$, mayor número de hijos $(p=0.0001)$, mayor número de cabras y sementales $(p=0.03)$ y más horas dedicadas al pastoreo $(p=0.02)$.

Por otra parte, las características comunes entre los grupos incluyen que entre el 50 y $100 \%$ de los productores complementan sus ingresos, ya sea por otros empleos, pensiones o apoyos por parte de familiares (estos apoyos son remesas que envían los familiares radicados en Estados Unidos principalmente). Al respecto, Kumar et al. (2015), señalan que el 51\% de los productores en el oeste de Uttar Pradesh, India, obtienen sus ingresos únicamente por la venta de leche, y el resto de los productores complementa sus ingresos con alguna actividad complementaria. Cuatro de los 5 grupos manifiestan poseer animales criollos, y sólo un grupo posee animales cruzados 
de las razas Nubia y Alpino. A este respecto, Montaldo et al. (2010) señalan que los productores poseen cabras que ellos llaman criollas; sin embargo, esta denominación es ahora aceptada como cabras locales. En este sentido, estudios como los de Maldonado-Jáquez et al. (2018) y Fonseka et al. (2018b), señalan que la mayoría de los productores poseen cabras locales en el norte de México y en Sri-Lanka, respectivamente. Los productores no llevan registros productivos y aquellos que llevan registros, sólo lo hacen de manera parcial, ya que sólo se registran de cabras preñadas y los abortos. Por lo anterior, para mejorar las características de los procesos de innovación, se debe recurrir a la motivación e información constante para incrementar la adopción de sistemas de registros entre los caprinocultores (Okkyla et al., 2014).

Tabla 3. Media \pm desviación estándar de las variables utilizadas en el ACP para la agrupación de caprinocultores del noreste de la Comarca Lagunera, en el estado de Coahuila

\begin{tabular}{|c|c|c|c|c|c|c|}
\hline Variable & $\begin{array}{c}\text { Grupo } 1 \\
(n=5)\end{array}$ & $\begin{array}{c}\text { Grupo } 2 \\
(n=9)\end{array}$ & $\begin{array}{c}\text { Grupo } 3 \\
(n=10)\end{array}$ & $\begin{array}{c}\text { Grupo } 4 \\
(n=4)\end{array}$ & $\begin{array}{c}\text { Grupo } 5 \\
(n=4)\end{array}$ & Valor $p$ \\
\hline Edad & $36.4 \pm 5.0^{b}$ & $36.1 \pm 8.2^{b}$ & $59.6 \pm 6.9^{a}$ & $76 \pm 8.5^{a}$ & $59 \pm 9.8^{a}$ & $<.0001$ \\
\hline Escolaridad & $2 \pm 0.7^{a b}$ & $2.4 \pm 0.5^{\mathrm{a}}$ & $0.9 \pm 0.9^{b c}$ & $0^{c}$ & $1 \pm 0.8^{b c}$ & 0.0002 \\
\hline $\begin{array}{l}\text { Número de } \\
\text { hijos }\end{array}$ & $1.6 \pm 1.1^{b}$ & $2.1 \pm 1.1^{b}$ & $3.8 \pm 1.1^{a}$ & $0^{\mathrm{b}}$ & $5.5 \pm 1.7^{a}$ & $<.0001$ \\
\hline $\begin{array}{l}\text { Número de } \\
\text { dependientes }\end{array}$ & $3.6 \pm 3.5$ & $3.1 \pm 1.1$ & $3.6 \pm 1.6$ & $4.5 \pm 0.7$ & $3.3 \pm 1.5$ & 0.8973 \\
\hline $\begin{array}{l}\text { Años en la } \\
\text { actividad }\end{array}$ & $21.4 \pm 8.9^{b}$ & $18.3 \pm 9.8^{b}$ & $29.8 \pm 16.2^{b}$ & $66.5 \pm 9.2^{\mathrm{a}}$ & $28.3 \pm 11.2^{b}$ & 0.0011 \\
\hline $\begin{array}{l}\text { Número de } \\
\text { cabras }\end{array}$ & $107.8 \pm 40.4$ & $74.2 \pm 31.4$ & $69.9 \pm 35.9$ & $74.5 \pm 13.4$ & $127.5 \pm 43.5$ & 0.0511 \\
\hline $\begin{array}{l}\text { Número de } \\
\text { sementales }\end{array}$ & $2.8 \pm 0.8^{\mathrm{ab}}$ & $2 \pm 1^{a b}$ & $1.4 \pm 0.7^{b}$ & $1.5 \pm 0.7^{a b}$ & $3.3 \pm 1.9^{a}$ & 0.0198 \\
\hline $\begin{array}{l}\text { Horas de } \\
\text { pastoreo }\end{array}$ & $7.6 \pm 2.2^{b}$ & $8 \pm 2.2^{b}$ & $6.1 \pm 2.5^{b}$ & $6^{b}$ & $10.8 \pm 2.5^{a}$ & 0.0236 \\
\hline $\begin{array}{l}\text { Litros de leche } \\
\text { cabra }^{-1}\end{array}$ & $2.0 \pm 0.5^{\mathrm{a}}$ & $1.6 \pm 0.5^{\mathrm{ab}}$ & $1.4 \pm 0.6^{\mathrm{ab}}$ & $0.5^{\mathrm{b}}$ & $1.5 \pm 0.4^{\mathrm{ab}}$ & 0.0308 \\
\hline $\begin{array}{l}\text { Precio por litro } \\
\text { de leche* }\end{array}$ & $0.2 \pm 0.1$ & $0.2 \pm 0.1$ & $0.3 \pm 0.1$ & $0.2 \pm 0.1$ & $0.2 \pm 0.1$ & 0.3205 \\
\hline $\begin{array}{l}\text { Hectáreas } \\
\text { totales }\end{array}$ & $5.1 \pm 3.0^{a}$ & $0.3 \pm 0.5^{b}$ & $2 \pm 3.1^{a b}$ & $4.5 \pm 0.7^{a b}$ & $4.4 \pm 3.8^{\mathrm{ab}}$ & 0.0155 \\
\hline $\begin{array}{l}\text { Hectáreas } \\
\text { sembradas }\end{array}$ & $4.7 \pm 2.2^{a}$ & $0.2 \pm 0.4^{b}$ & $0.3 \pm 0.5^{b}$ & $0.5 \pm 0.7^{b}$ & $1.4 \pm 1.1^{\mathrm{b}}$ & $<.0001$ \\
\hline $\begin{array}{l}\text { Años en el } \\
\text { domicilio actual }\end{array}$ & $26.4 \pm 14.7$ & $19.7 \pm 14.3$ & $32.6 \pm 22.3$ & $55 \pm 21.2$ & $30.8 \pm 20.2$ & 0.1936 \\
\hline
\end{tabular}

D.E.= Desviación Estándar. abc Diferente literal entre líneas indican diferencias $(p<0.05) .{ }^{*}$ Precio por tipo de cambio de $\$ 1.00$ dólares americanos por $\$ 19.49 \mathrm{MXN}$ al 8 de mayo de 2019)

Los productores dedican entre 6 y 10 horas al pastoreo por día; y la producción de leche oscila entre los 500 y los $2000 \mathrm{gr}$, la cual se vende entre $\$ 0.21$ y $\$ 0.26$ Dólares/litro. La mayoría de productores no almacenan alimento para las épocas críticas de alimentación, no cuentan con asistencia técnica la mayoría de ellos, no han sido capacitados; sólo un bajo porcentaje ha recibido algún tipo de capacitación (8 al $11 \%$ ) y han aprendido a elaborar quesos, dulces, chorizo, entre otros. Al respecto, 
Alva-Pérez et al. (2018), señalan que caprinocultores de Tamaulipas, México, dedican alrededor de 7 horas al pastoreo y las cabras producen $680 \mathrm{ml}$ de leche por día, lo cual coincide parcialmente con nuestros resultados. Sandhu et al. (2018), señalan que el $65 \%$ de los productores en Punjab, India, no conservan forraje. Hassan et al. (2016), refieren que productores de bajos recursos poseen un inadecuado conocimiento sobre la prevención y tratamiento de enfermedades, por lo que requieren de asistencia técnica. De igual manera, Vatta et al. (2010), señalan que la capacitación constante brinda la oportunidad de facilitar la adquisición de habilidades a los pequeños caprinocultores.

\section{Principales Limitantes}

Respecto a los resultados obtenidos en el taller realizado con los productores entrevistados previamente, en la tabla 4, se presenta un extracto del árbol de problemas general, y donde la percepción de los productores sobre las principales limitantes a la producción se centran en tres problemas: 1. Riesgo de disminuir la producción de leche por falta de alimento; 2. Pérdida de interés en la actividad por futuras generaciones; y 3. Riesgo en la producción de leche por deficiencia en manejo sanitario de los animales. Los cuales, de no resolverse, pondrían en riesgo la productividad de todo el sistema de producción de leche, y en consecuencia la principal fuente de ingresos de numerosas familias en la Comarca Lagunera. Al respecto, estos resultados son similares a los encontrados con el reporte de Raja et al. (2018), en donde caprinocultores de la India señalan limitantes similares a las encontradas en este estudio. Por otra parte, Hassan et al. (2016) señalan que una de las principales preocupaciones de los caprinocultores de Bangladesh se centra en el status sanitario de las explotaciones. Esta información sugiere que la problemática que enfrentan los productores caprinos en las zonas áridas y semiáridas del mundo es similar. Sin embargo, para el diseño de un plan estratégico adecuado a las circunstancias de productores, se hace necesario que los objetivos y acciones estratégicos sean acordes a las causas raíz de su problemática, y las variables de respuesta de mayor peso.

Tabla 4. Principales problemas que afectan al sistema de producción caprino según la perspectiva de los productores de la porción noroeste de la Región Lagunera de Coahuila, en el norte de México.

\begin{tabular}{|c|c|c|c|}
\hline $\begin{array}{c}\text { Problemática } \\
\text { principal }\end{array}$ & & Causas & Efectos \\
\hline \multirow{2}{*}{$\begin{array}{c}\text { En riesgo alta } \\
\text { producción de leche } \\
\text { por déficit alimenticio y } \\
\text { un plan de } \\
\text { mejoramiento genético }\end{array}$} & $\begin{array}{l}\text { Falta de una } \\
\text { estrategia de } \\
\text { mejoramiento } \\
\text { genético }\end{array}$ & $\begin{array}{c}\text { Falta de registros de } \\
\text { producción } \\
\text { Compra de animales de } \\
\text { reemplazo a terceros } \\
\text { Falta de esquemas de } \\
\text { cruzamientos }\end{array}$ & $\begin{array}{c}\text { Pérdida por } \\
\text { desconocimiento de la } \\
\text { genética de las mejores } \\
\text { cabras }\end{array}$ \\
\hline & $\begin{array}{l}\text { Inestabilidad y } \\
\text { dependencia de } \\
\text { alimento en } \\
\text { áreas de cultivo }\end{array}$ & $\begin{array}{l}\text { Afectación a cultivos por } \\
\text { efecto del cambio climático } \\
\text { Sobre pastoreo }\end{array}$ & $\begin{array}{l}\text { Déficit en la producción de } \\
\text { forrajes } \\
\text { Reducción de la } \\
\text { producción por no ofrecer }\end{array}$ \\
\hline
\end{tabular}




\begin{tabular}{|c|c|c|c|}
\hline & & $\begin{array}{c}\text { Pocos recursos para } \\
\text { complementar al ganado }\end{array}$ & $\begin{array}{c}\text { un complemento después } \\
\text { del Pastoreo }\end{array}$ \\
\hline \multirow{6}{*}{$\begin{array}{c}\text { Pérdida de interés en la } \\
\text { actividad caprina por } \\
\text { las nuevas } \\
\text { generaciones y } \\
\text { limitadas opciones de } \\
\text { mercado por falta de } \\
\text { atención en la calidad } \\
\text { de la leche }\end{array}$} & \multirow{5}{*}{$\begin{array}{l}\text { Falta de visión } \\
\text { como negocio } \\
\text { familiar y } \\
\text { migración a } \\
\text { nuevos modelos } \\
\text { de negocio }\end{array}$} & $\begin{array}{c}\text { Falta de conocimiento } \\
\text { sobre productos con valor } \\
\text { agregado }\end{array}$ & $\begin{array}{l}\text { No hay innovación en la } \\
\text { creación de nuevos } \\
\text { productos }\end{array}$ \\
\hline & & $\begin{array}{l}\text { Productores de mayor } \\
\text { edad }\end{array}$ & $\begin{array}{l}\text { Precio de litro de leche } \\
\text { establecido por el } \\
\text { intermediario }\end{array}$ \\
\hline & & $\begin{array}{l}\text { Venta de leche como } \\
\text { única fuente de ingreso }\end{array}$ & Baja producción de leche \\
\hline & & $\begin{array}{l}\text { Se requiere complementar } \\
\text { el ingreso con otras } \\
\text { fuentes de empleo }\end{array}$ & Falta de recurso humano \\
\hline & & $\begin{array}{c}\text { Falta de inversión en la } \\
\text { actividad }\end{array}$ & $\begin{array}{l}\text { Se compromete la } \\
\text { principal fuente de } \\
\text { ingresos }\end{array}$ \\
\hline & $\begin{array}{c}\text { Baja calidad en } \\
\text { la leche de cabra }\end{array}$ & $\begin{array}{l}\text { Falta de áreas específicas } \\
\text { para ordeñar } \\
\text { Falta de manejo adecuado } \\
\text { de leche post ordeña }\end{array}$ & $\begin{array}{l}\text { Sin acceso a nuevos } \\
\text { mercados }\end{array}$ \\
\hline \multirow{3}{*}{$\begin{array}{l}\text { Principal fuente de } \\
\text { ingresos comprometida } \\
\text { por manejo deficiente } \\
\text { de la salud animal }\end{array}$} & $\begin{array}{l}\text { Recursos } \\
\text { económicos }\end{array}$ & $\begin{array}{l}\text { Poca o nula asistencia } \\
\text { médica veterinaria }\end{array}$ & Alta incidencia de abortos \\
\hline & $\begin{array}{l}\text { cuidado de la } \\
\text { salud animal }\end{array}$ & $\begin{array}{l}\text { Escasos recursos para } \\
\text { programas sanitarios }\end{array}$ & Reducción del hato \\
\hline & $\begin{array}{l}\text { Falta de recursos } \\
\text { para mejorar la } \\
\text { infraestructura }\end{array}$ & $\begin{array}{c}\text { No se separan animales } \\
\text { enfermos }\end{array}$ & $\begin{array}{l}\text { Baja productividad de } \\
\text { leche }\end{array}$ \\
\hline
\end{tabular}

Fuente: Elaboración propia

\section{Estrategias para el desarrollo de los productores}

La caprinocultura es una opción importante para manutención de productores rurales de escasos recursos, y se trata de la principal actividad económica pecuaria en las áreas desérticas y semidesérticas de México (Alva-Pérez et al., 2018); por ello, en la tabla 5 se muestran las estrategias sugeridas para impulsar el desarrollo de los productores, mismas que se plantean con base al resultado obtenido en el ACJ (figura 1), del ACM (figura 2), y del análisis de causa-efecto del árbol de problemas (tabla 4). Para cada grupo o conglomerado de productores dentro de la Cooperativa, se plantea un objetivo estratégico y acciones a implementar para resolver la principal problemática de cada conglomerado (tabla 5). Asimismo, cada objetivo y acción estratégica se diseñó según la perspectiva y variables a mejorar a las especificidades del conjunto de productores de cada conglomerado. Por lo que, dentro del plan estratégico se definieron acciones de innovación, capacitación y/o transferencia de tecnología; las cuales son producto de la demanda de los mismos productores y se complementan con experiencias previas reportadas en la literatura científica.

Esta información coincide con la literatura existente para pequeños productores en México. Al respecto, Cárdenas-Bejarano et al. (2016), señalan que el índice de 
adopción de tecnologías incrementa en la medida que los productores se organizan; ya que hay un mejor flujo de información al interior de los grupos e indican que para entender mejor los procesos de adopción tecnológica, es necesario considerar el análisis de la estructura del grupo al que pertenecen; así como las interrelaciones existentes sin olvidar el contexto socioeconómico. Por otra parte, algunos otros autores refieren algunas sugerencias $\mathrm{y} / \mathrm{o}$ recomendaciones para resolver problemáticas similares a las encontradas en los grupos de productores de este estudio; por ejemplo, existen reportes (Tanwar et al., 2011; Fonseka et al.,2018b), que sugieren desarrollar esquemas de conservación de alimento y de conservación de material genético sobresaliente a través de la selección, así como la interacción entre los diferentes actores de la cadena de producción para facilitar el flujo de información de mercados y el desarrollo de infraestructura que mejore el sistema de marketing para leche de cabra (Chipasha et al.,2017); o el entrenamiento de los integrantes y organización para mejorar la productividad y la sanidad de las unidades de producción (Hundal et al., 2016; Kumar et al., 2015; Sandhu et al., 2018); así como el acceso a crédito y servicios de extensión como detonantes para el éxito de las empresas dedicadas a la producción de cabras (Ifeanyichukwe et al., 2018). Además, si se considera lo que señala Barrera-Perales et al. (2018), algunos aspectos que juegan a favor de los productores del semiárido mexicano, es que el uso de mano de obra exclusivamente familiar, bajo nivel tecnológico, infraestructura limitada y dependencia del agostadero; son factores que abonan a la rentabilidad y continuidad de las unidades de producción, ya que no se requieren grandes inversiones para operar, y esto a su vez ayuda a paliar los efectos limitantes del sistema de producción actual.

Tabla 5. Acciones estratégicas para el desarrollo de los productores por conglomerado para la gestión de la innovación

\begin{tabular}{|c|c|c|c|c|c|}
\hline Grupo & Descripción & $\begin{array}{l}\text { Problema } \\
\text { Principal }\end{array}$ & Objetivo & $\begin{array}{l}\text { Problemas } \\
\text { Específicos } \\
\text { (Causas Raíz) }\end{array}$ & $\begin{array}{l}\text { Acciones } \\
\text { Estratégicas }\end{array}$ \\
\hline 1 & $\begin{array}{c}\text { >producción de } \\
\text { leche (2.4 L/día); } \\
\text { > sup. de cultivo } \\
\text { (4.7 ha); >\# de } \\
\text { cabras (107/UP); } \\
\text { Transición a } \\
\text { sistema semi- } \\
\text { extensivo }\end{array}$ & $\begin{array}{l}\text { En riesgo la } \\
\text { alta } \\
\text { producción de } \\
\text { leche de } \\
\text { cabra por } \\
\text { déficit } \\
\text { alimenticio }\end{array}$ & $\begin{array}{l}\text { Asegurar la } \\
\text { alta } \\
\text { producción de } \\
\text { leche de } \\
\text { cabra }\end{array}$ & $\begin{array}{l}\text { a. Afectación de } \\
\text { cultivos y pérdida } \\
\text { de residuos de } \\
\text { cosecha, plagas, } \\
\text { inundaciones, } \\
\text { sequía. } \\
\text { b. Carencia de } \\
\text { mejoramiento } \\
\text { genético caprino }\end{array}$ & $\begin{array}{l}\text { Innovación y } \\
\text { capacitación en } \\
\text { conservación } \\
\text { de residuos de } \\
\text { cosecha. } \\
\text { Selección de } \\
\text { cabras con } \\
\text { mayor } \\
\text { producción }\end{array}$ \\
\hline 2 & $\begin{array}{c}>\text { Grado } \\
\text { académico; } 56 \% \\
\text { de jefes de familia } \\
\text { mujeres; } \\
\text { Producción leche } \\
\text { (1.56 L/cabra); } \\
\text { Jóvenes deseo } \\
\text { estudiar } \\
\text { universidad; } \\
\text { Venta de leche de } \\
\text { cabra coadyuva: } \\
\text { manutención }\end{array}$ & $\begin{array}{l}\text { a. Pérdida de } \\
\text { interés en la } \\
\text { actividad } \\
\text { caprina por } \\
\text { las nuevas } \\
\text { generaciones } \\
\\
\text { b. Asegurar la } \\
\text { producción de } \\
\text { cultivo. }\end{array}$ & $\begin{array}{l}\text { a. Fomentar } \\
\text { el interés por } \\
\text { la } \\
\text { caprinocultura } \\
\text { a nuevas } \\
\text { generaciones. } \\
\\
\text { b. Gestión de } \\
\text { recursos para } \\
\text { financiar y } \\
\text { proteger } \\
\text { cultivos. }\end{array}$ & $\begin{array}{l}\text { a. Falta de visión } \\
\text { de modelo de } \\
\text { negocio familiar. } \\
\text { b. } \\
\text { Desconocimiento } \\
\text { de valor } \\
\text { agregado a la } \\
\text { leche. } \\
\text { c. Falta de } \\
\text { inocuidad en la }\end{array}$ & $\begin{array}{l}\text { Desarrollo de } \\
\text { nuevos } \\
\text { modelos de } \\
\text { negocios e } \\
\text { implementación } \\
\text { de buenas } \\
\text { prácticas de } \\
\text { ordeño y } \\
\text { manejo de la } \\
\text { leche. }\end{array}$ \\
\hline
\end{tabular}




\begin{tabular}{|c|c|c|c|c|c|}
\hline & $\begin{array}{l}\text { ganado, sustento } \\
\text { hogar, educación }\end{array}$ & & & $\begin{array}{l}\text { producción de } \\
\text { leche de cabra }\end{array}$ & \\
\hline 3 & $\begin{array}{c}\text { Producción de } \\
\text { leche (1.38 L/día); } \\
75 \% \text { de las } \\
\text { cabras ha } \\
\text { presentado } \\
\text { aborto; } 75 \% \text { de } \\
\text { productores no } \\
\text { cuenta asistencia } \\
\text { veterinaria; } \\
\text { Nuevas } \\
\text { generaciones } \\
\text { interesadas en } \\
\text { producción } \\
\text { caprina }\end{array}$ & $\begin{array}{l}\text { Principal } \\
\text { fuente de } \\
\text { ingresos } \\
\text { comprometida } \\
\text { por la salud } \\
\text { animal y falta } \\
\text { de buenas } \\
\text { prácticas de } \\
\text { ordeña de las } \\
\text { cabras }\end{array}$ & $\begin{array}{c}\text { Gestionar } \\
\text { apoyos } \\
\text { financieros y } \\
\text { alianzas para } \\
\text { consultoría } \\
\text { con } \\
\text { universidades } \\
\text { de veterinaria }\end{array}$ & $\begin{array}{c}\text { Recursos } \\
\text { económicos } \\
\text { limitados para el } \\
\text { cuidado de la } \\
\text { salud animal y } \\
\text { mejora de } \\
\text { corrales de } \\
\text { encierro. } \\
\text { Falta de higiene } \\
\text { personal durante } \\
\text { la ordeña. }\end{array}$ & $\begin{array}{l}\text { Gestión de } \\
\text { recursos para } \\
\text { financiar y } \\
\text { mejorar la } \\
\text { infraestructura } \\
\text { de los corrales. } \\
\text { Alianza } \\
\text { estratégica con } \\
\text { instituciones } \\
\text { para mejorar la } \\
\text { salud de los } \\
\text { rebaños }\end{array}$ \\
\hline 4 & $\begin{array}{c}\text { Baja producción } \\
\text { (0.5 L/cabra); } \\
\text { Precio por litro de } \\
\text { leche bajo ( } \$ 0.20 \\
\text { Dlls/L); Sin } \\
\text { integración } \\
\text { familiar; } \\
\text { Mayor edad del } \\
\text { productor (76 } \\
\text { años). }\end{array}$ & $\begin{array}{c}\text { Baja } \\
\text { producción de } \\
\text { leche de } \\
\text { cabra por } \\
\text { falta de } \\
\text { alimento y } \\
\text { personal de } \\
\text { apoyo }\end{array}$ & $\begin{array}{c}\text { Gestionar } \\
\text { alianzas con } \\
\text { otros rebaños } \\
\text { de la } \\
\text { cooperativa. }\end{array}$ & $\begin{array}{c}\text { a. Sobre } \\
\text { explotación de } \\
\text { las tierras de } \\
\text { pastoreo. } \\
\text { b. No se } \\
\text { garantiza el } \\
\text { suministro de } \\
\text { agua limpia. } \\
\text { Déficit } \\
\text { alimentario }\end{array}$ & $\begin{array}{c}\text { Definir } \\
\text { contratos de } \\
\text { cooperación } \\
\text { con otros } \\
\text { productores. } \\
\text { Integración los } \\
\text { productores } \\
\text { mayores en la } \\
\text { capacitación en } \\
\text { acción }\end{array}$ \\
\hline 5 & $\begin{array}{l}\text { Producción media } \\
1.5 \text { L/cabra de } \\
\text { leche al día. } \\
\text { Mayor número de } \\
\text { cabras (125) por } \\
\text { rebaño. Mayor } \\
\text { número de hijos. } \\
\text { Sistema extensivo } \\
10 \text { horas pastoreo } \\
\text { al día. Dependen } \\
\text { de mezquite para } \\
\text { complementar la } \\
\text { alimentación del } \\
\text { rebaño. }\end{array}$ & $\begin{array}{l}\text { En riesgo la } \\
\text { producción de } \\
\text { leche de } \\
\text { cabra por } \\
\text { falta de } \\
\text { alimento y } \\
\text { tierras propias }\end{array}$ & $\begin{array}{l}\text { Gestión de } \\
\text { recursos para } \\
\text { financiar y } \\
\text { mejorar la } \\
\text { infraestructura } \\
\text { de los } \\
\text { corrales. }\end{array}$ & $\begin{array}{c}\text { Rentan tierras de } \\
\text { cultivo. Solo el } \\
50 \% \text { de los } \\
\text { productores tiene } \\
\text { alimento contra } \\
\text { contingencias. }\end{array}$ & $\begin{array}{c}\text { Conservación } \\
\text { de residuos de } \\
\text { cosecha. } \\
\text { Elaboración de } \\
\text { bloques } \\
\text { nutricionales. } \\
\text { Conservación } \\
\text { de vainas de } \\
\text { mezquite }\end{array}$ \\
\hline
\end{tabular}

\section{CONCLUSIONES}

Los resultados encontrados sugieren que el delimitar y discriminar productores rigurosamente de acuerdo a sus similitudes, así como la identificación de limitantes en el sistema de producción caprino, desde el punto de vista de los productores, permite estratificarlos y agruparlos de manera que se pueden identificar claramente los puntos críticos de atención para el establecimiento de esquemas de investigación, innovación y transferencia de tecnología, acordes a la realidad de los mismos. Esto permitirá en el futuro inmediato, que la definición de estrategias y políticas públicas de fortalecimiento a los sistemas de producción caprinos, tanto intensivos como extensivos de las zonas áridas y semiáridas de México, impacten eficientemente; y se consolide la caprinocultura del norte de nuestro país, como una actividad generadora de empleos en áreas marginales. 


\section{LITERATURA CITADA}

AGUDELO-GÓMEZ DA, Pelicioni-Savegnago R, Buzanskas ME, Ferraudo AS, Prado Munari D, Cerón-Muñoz MF. 2015. Genetic principal components for reproductive and productive traits in dual-purpose buffaloes in Colombia. Journal of Animal Science. 93: 3801-3809. https://doi.org/10.2527/jas.2015-8940

AGUILAR A, Cabral A, Alvarado T. 2016. La Técnica del Proceso Administrativo Agropecuario Estratégico - PAAE Versión 2016. Revista Mexicana de Agronegocios. 20(38): 209-216. https://www.redalyc.org/pdf/141/14146082011.pdf

ALVA-PÉREZ J, López-Corona LE, Zapata-Campos CC, Vázquez-Villanueva J, Barrios-García HB. 2018. Condiciones productivas y zoosanitarias de la producción caprina en el altiplano de Tamaulipas, México. Interciencia. 44(3): 154-160. https://www.redalyc.org/jatsRepo/339/33958848008/33958848008.pdf

BALESTRI L, Ferrán A, Giorgis A, Saravia C, Larrea A, Castaldo A, Poma K, Pariani A. 2001. La toma de decisiones en las empresas agropecuarias del norte de la provincia de la Pampa. Ciencia Veterinaria. Facultad de Ciencias Veterinarias. UNLPam. 3(1):113-129.

https://cerac.unlpam.edu.ar/index.php/veterinaria/article/view/1998/1954

BALZARINI MG, González L, Tablada M, Casanoves F, Di Rienzo JA, Robledo CW. 2008. Manual del Usuario. Editorial Brujas. Córdoba, Argentina. https://www.researchgate.net/publication/283491340_Infostat_manual_del_usuario

BARRERA-PERALES OT, Sagarnaga-Villegas LM, Sala-González JM, LesoRodríguez JA, Santos-Lavalle R. 2018. Viabilidad económica de la ganadería caprina en San Luis Potosí, México. Mundo Agrario. 19(40): https://doi.org/10.24215/15155994e077

BERNAL-GARCÍA J, Martínez MS, Sánchez-García J. 2003. Modelización de los factores más importantes que caracterizan un sitio en la red. XII Jornadas de ASEPUMA. Universidad de Murcia, España. 16 y 17 de septiembre de 2004. Pp: 113. https://www.um.es/asepuma04/resumen/resumen_bernal_martinez_sanchez.pdf

CÁRDENAS-BEJARANO E, Gallardo-López F, Núñez-Espinoza JF, Asiaín-Hoyos A, Rodríguez-Chessani MA, Velázquez-Beltrán LG. 2016. Redes de innovación en los grupos ganaderos de validación de transferencia de tecnología en México. Agricultura, Sociedad y Desarrollo. 13: 237-255. http://www.scielo.org.mx/pdf/asd/v13n2/18705472-asd-13-02-00237.pdf

CARRASCO-GONZÁLEZ A. 2004. Factores psicosociales y comportamientos de salud relacionados con el consumo de alcohol en adolecentes: un análisis multivariable. Revista Latinoamericana de Psicología. 36(1): 125-144. https://www.redalyc.org/pdf/805/80536111.pdf

CASTEL J, Mena Y, Delgado M, Camúñez J, Basulto J, Cavanca F, Guzmán J, Alcalde M. 2003. Characterization of semi-extensive goat production systems in southern 
Spain. Small Ruminant Research. 47: 133-143. https://doi.org/10.1016/S09214488(02)00250-X

CHIPASHA H, Ariyawardana A, Mortlock MY. 2017. Smallholder goat farmer's market participation in Choma district, Zambia. African Journal of Food, Agriculture, Nutrition and Development. 17(1): 11691-708.

http://ajfand.net/Volume17/No1/Chipasha16175.pdf

COFRÉ P. 2001. Producción de Cabras Lecheras. Instituto de Investigaciones Agropecuarias. Chillán, Chile. Boletín INA. No.66. Pp. 200.

DUBEUF J. 2005. Structural, market and organizational conditions for developing goat dairy production systems. Small Ruminant Research. 60: 67-74. https://doi.org/10.1016/j.smallrumres.2005.06.015

ESCAREÑO L, Salinas-González H, Wurzinger M, Iñiguez L, Sölkner J, Meza-Herrera C. 2012. Dairy goat production systems. Tropical Animal Health and Production. 45: 17-34. https://doi.org/10.1007/s11250-012-0246-6

ESCAREÑO SL, Wurzinger M, Pastor-López F, Salinas H, Johan S, Iñiguez L. 2011. La cabra y los sistemas de producción caprina de los pequeños productores de la Comarca Lagunera, en el norte de México. Rev. Chapingo Serie Ciencias Forestales y del Ambiente. 17: 235-246. http://dx.doi.org/10.5154/r.rchscfa.2010.10.087

ESCOBAR J, Bonilla-Jiménez FI. 2009. Grupos focales: Una guía conceptual y metodológica. Cuadernos Hispanoamericanos de Psicología. 9(1): 51-67. http://biblioteca.udgvirtual.udg.mx:8080/jspui/bitstream/123456789/957/1/Gupos\%20f ocales\%20una\%20gu\%c3\%ada\%20conceptual\%20y\%20metodol\%c3\%b3gica.pdf

FONSEKA WS, Mahusoon MM, Narmhikaa K, Inthujaa S. 2018a. Socio economic status and infrastructure availability of goat farmers in Eravur and Vantharumoolai Veterinary Ranges in Batticaloa district. International Journal of research Publications. 7(1). http://ijrp.org/paper_detail/268

FONSEKA WS, Mahusoon MM, Narmhikaa K. 2018b. The rearing system of goats in Mahaoya Veterinary Range in Ampara district, Sri Lanka. International Research $\begin{array}{llll}\text { Journal of } \quad \text { Biological } & \text { 26-31. }\end{array}$ http://www.isca.in/IJBS/Archive/v7/i12/5.ISCA-IRJBS-2018-068.pdf

GARCÍA-PÉREZ S. 2017. Las Empresas Agropecuarias y la Administración Financiera. Revista Mexicana de Agronegocios. 40: 583-594. https://www.redalyc.org/jatsRepo/141/14152127007/html/index.html

GÓMEZ RJ. 2007. La caprinocultura como elemento articulador del desarrollo rural en el altiplano potosino. Tesis Doctoral. Universidad Autónoma de San Luis Potosí. San Luis Potosí, México. http://ninive.uaslp.mx/xmlui/handle/i/2061

GUERRA G. 1998. Manual de Administración de Empresas Agropecuarias. 2ª Edición. Editorial Agroamérica. San José, Costa Rica. ISBN 92-9039-181-2 
GUERRERO-CRUZ M. 2010. La caprinocultura en México, una estrategia de desarrollo. Revista Universitaria Digital de Ciencias Sociales. 1(1): 1-7. http://virtual.cuautitlan.unam.mx/rudics/?p=403

HASSAN MM, Shaeef Z, Alam M, Hossain ME, Islam S, Uddin MB. 2016. Perception of smallholding goat farmers on disease conditions of goat in Bangladesh. International Journal of Natural Sciences. 6(1): 43-48. https://www.researchgate.net/publication/320417063_Perception_of_Smallholding_G oat_Farmers_on_Disease_conditions_of_Goat_in_Bangladesh

HERNÁNDEZ-HERNÁNDEZ N, Garnica-González J. 2015. Árbol de Problemas del análisis al diseño y desarrollo de productos. Conciencia Tecnológica. 50: 38-46. https://www.redalyc.org/pdf/944/94443423006.pdf

HERNÁNDEZ-SAMPIERI R Fernández-Collado C, Baptista-Lucio P. 2006. Metodología de la investigación. Quinta edición. México: McGraw-Hill Interamericana. https://www.esup.edu.pe/descargas/dep_investigacion/Metodologia\%20de\%20la\%20 investigaci\%C3\%B3n\%205ta\%20Edici\%C3\%B3n.pdf

HUNDAL JS, Singh U, Singh N, Kansal SK, Bhatti JS. 2016. Impact of training on knowledge level of goat farmers in Punjab. Haryana Vet. 55(1): 47-49. https://www.luvas.edu.in/haryana-veterinarian/download/harvet2016/10.pdf

IFEANYICHUKWE US, Ezeano Cl, Anozie R. 2018. Climate change adaptation coping strategies among sheep and goat farmers in Ivo local government area of Ebonyi state, Nigeria. Sustainability, Agri, Food and Environmental Research. 6(2): 50-68. https://www.researchgate.net/publication/327670176_Climate_change_and_Adaptati on_Coping_Strategies_among_Sheep_and_Goat_Farmers_in_Ivo_Local_Governme nt_Area_of_Ebonyi_State_Nigeria

INAFED. Instituto Nacional para el Federalismo y Desarrollo Municipal. 2010. Enciclopedia de los municipios y delegaciones de México. http://www.inafed.gob.mx/work/enciclopedia/EMM05coahuila/.

KUMAR S, Chauhan HS, Kide W, Mayekar AJ. 2015. Socio-economic profile of goat farmers in Western Uttar Pradesh (India). International Conference on Agriculture, Veterinary and Environmental Science. Organized by International Multidisciplinary Research Foundation, held At: Manorma Hotel Vijayawada, Andhra Pradesh India 2526 July, 2015. 2(1): 43-46.

https://www.researchgate.net/publication/299510803_SOCIO-

ECONOMIC_PROFILE_OF_GOAT_FARMERS_IN_WESTERN_UTTAR_PRADESH INDIA

MALDONADO-JÁQUEZ JA, Salinas-González H, Torres-Hernández G, Becerril-Pérez CM, Díaz-Rivera P. 2018. Factors influencing milk production of local goats in the Comarca Lagunera, México. Livestock Research for Rural Development. 30. No. Art. 32. http://www.Irrd.org//rrd30/7/glat30132.html 
MENDOZA-JIMENEZ A, Ortega-Sánchez JL. 2009. Caracterización de la caprinocultura en el municipio de Tepelmeme, Villa de Morelos, Oaxaca, México. Revista Chapingo Serie Zonas Áridas. 8(1): 75-80. https://www.redalyc.org/pdf/4555/455545064011.pdf

MONTALDO HH, Torres-Hernández G, Valencia-Posadas M. 2010. Goat breeding research in Mexico. Small Ruminant Research. 89: 155-163. https://doi.org/10.1016/j.smallrumres.2009.12.039

OKKYLA S, Dwijatmiko S, Kurnianto E. 2014. Path analysis of recording system innovation factors affecting adoption of goat farmers. Journal of the Indonesian Tropical Animal Agriculture. 39(1): 58-64. https://doi.org/10.14710/jitaa.39.1.58-64

PEÑA-MALAVERA A, Bruno C, Teich I, Fernandez E, Balzarini 2010. Análisis de conglomerados en la identificación de estructura genética a partir de datos de marcadores moleculares. Tumbaga. 1(5):225-237. https://dialnet.unirioja.es/servlet/articulo?codigo=3628389

RAJA T, Prabu M, Serma Saravana Pandian A, Thirunavukkarasu P. 2018. Factors influencing the constraints perceived by the small scale backyard goat farmers. International Journal of Livestock Research. 8(12): 175-181. https://www.ejmanager.com/mnstemps/68/68-1525773470.pdf?t=1587477289

SAGARPA. Secretaria de Agricultura, Ganadería Recursos Naturales, Pesca y Alimentación. 2007. Programa Nacional Pecuario 2007-2012. http://www.sagarpa.gob.mx/ganaderia/Publicaciones/Lists/Programa\%20Nacional\%2 OPecuario/Attachments/1/PNP260907.pdf

SAGARPA. Secretaria de Agricultura, Ganadería Recursos Naturales, Pesca y Alimentación. 2015. Cuestionario de evaluación al productor. Manual de buenas prácticas en producción de leche caprina. http://www.sagarpa.gob.mx/ganaderia/Publicaciones/Lists/Manuales\%20de\%20Buen as\%20Prcticas/Attachments/3/manual_cabra.pdf

SALINAS-GONZÁLEZ H, Echavarría FG, Flores-Nájera MJ, Flores-Ortíz MA, Gutiérrez R, Rumayor A, Meza-Herrera CA, Pastor-López FJ. 2011. Participatory evaluation of goat technologies in semiarid North Central México. Rev. Chapingo Ciencias Forestales y del Medio Ambiente. 27: 225-234. http://dx.doi.org/10.5154/r.rchscfa.2010.11.106

SALINAS-GONZÁLEZ H, Meza-Herrera CA, Escareño LM, Echavarría ChFG, Maldonado-Jáquez JA, Pastor-López FJ. 2015. Meat and milk production systems in goats: Productive trends in Mexico and the world, in: Díaz AE, Tórtora PJL, Palomares REG, Gutiérrez HJL (Eds.). Enfermedades de las Cabras, Libro Científico 1. INIFAP, México DF. Pp. 3-22. ISBN: 978-607-37-0411-3.

SALINAS-GONZÁLEZ H, Valle-Moysen E, Santiago-Miramontes M, Veliz-Deras F, Maldonado-Jáquez JA, Vélez-Monroy L, Torres-Hernández D, Isidro-Requejo L, 
Figueroa-Viramontes U. 2016. Análisis descriptivo de unidades caprinas en el suroeste de la Región Lagunera, Coahuila, México. Interciencia. 44(11): 763-768. https://www.redalyc.org/pdf/339/33948191006.pdf

SANDHU SS, Malik DS, Kaswan S, Singh J, Singh Y. 2018. Feeding management practices adopted by goat farmers in South West Punjab and their constraints. International Journal of Livestock Research. 8(5): 271-279. http://dx.doi.org/10.5455/ijlr.20170828080418

SANTIAGO J, Roussos A. 2010. El focus groups como técnica de investigación cualitativa. Documento de trabajo \#256. Universidad de Belgrano. Disponible en: http://www.ub.edu.ar/investigaciones/dt_nuevos/256_roussos.pdf

SANZ M, Fernández J, De la Torre G, Ramos E, Carmona F, Boza J. 2003. Calidad de la leche de los pequeños rumiantes. Anales de la Real Academia de Ciencias Veterinarias de Andalucía Oriental. 16(1): 155-166. http://racvao.es/wpcontent/uploads/2014/12/Calidad-de-la-leche.pdf

TANWAR PS. 2011. Constraints perceived by goat keepers in adoption of goat husbandry practices in semi-arid Rajasthan. Journal of Community Mobilization and Sustainable Development. 6(1): 108-111. https://www.researchgate.net/publication/326677491_Constraints_Perceived_by_Go at_Keepers_in_Adoption_of_Goat_Husbandry_Practices_in_Semi-Arid_Rajasthan

TORRES-Hernández G, Maldonado-Jáquez JA, Salinas-González H, Becerril-Pérez C: 2016. Efecto del número de mediciones de la producción de leche en la estimación de la curva de lactancia en cabras locales de la Comarca Lagunera, México. Revista de Sistemas 3(7):22-26. http://www.ecorfan.org/bolivia/researchjournals/Sistemas_Experimentales/vol3num7/ Revista_Sistemas_Experimentales_V3_N7_4.pdf

VATTA AF, de Villiers JF, Harrison LJS, Krecek RC, Pearson RA, Rijkenberg FHJ, Spickett A, Worth SH. 2010. Opportunities to train small-scale goat farmers within onfarm research projects. Advances in Animal Biosciences. 1(2): 504-504. https://doi.org/10.1017/S2040470010001202 